\title{
Téoros
}

Revue de recherche en tourisme

\section{La formation en tourisme au service des régions : l'expérience du Bas-Saint-Laurent/Gaspésie}

\section{Diane Caron, Isabelle Creusot et Alain Turcotte}

Volume 13, numéro 1, mars 1994

Bas-Saint-Laurent, Gaspésie, Iles-de-la-Madeleine : fascinantes

périphéries

URI : https://id.erudit.org/iderudit/1077772ar

DOI : https://doi.org/10.7202/1077772ar

Aller au sommaire du numéro

Éditeur(s)

Université du Québec à Montréal

ISSN

0712-8657 (imprimé)

1923-2705 (numérique)

Découvrir la revue

Citer cet article

Caron, D., Creusot, I. \& Turcotte, A. (1994). La formation en tourisme au service des régions : l'expérience du Bas-Saint-Laurent/Gaspésie. Téoros, 13(1), 24-28. https://doi.org/10.7202/1077772ar 


\section{La formation en tourisme au service des régions: I'expérience du Bas-Saint-Laurent/ Gaspésie \\ Diane Caron, Isabelle Creusot et Alain Turcotte"}

Quand on nous a proposé de collaborer au présent numéro, nous avons envisagé plusieurs scénarios de rédaction. Comment peut-onle mieux illustrer lacontributiond'une formation en tourisme dans une perspective de développement régional? Après müre réflexion, il nous est apparu évident que la meilleure façon de parler de formation, c'est à travers ses résultats, c'est-â-dire par ceuxlà mêmes qui exercent le métier avec professiannalisme.

Dans le but de couvrir l'ensemble des secteurs d'intervention touristique en région, nous avons sélectionné sept intervenants représentatifs de différentes sous-régions géographiques du Bas-Saint-Laurent/ Gaspésie et des divers niveaux de formation en tourisme.

Les entrevues ont porté sur les points suivants: a) description des tâches et responsabilités; b) utilité de la formation reçue avec illustration de problèmes qu"elle a permis de résoudre; cl vision présentée et future du tourisme dans la péninsule Bas-Saint-Laurent/ Gaspésie.

Les pages qui suivent sont faites d'extraits de ces entrevues. II ressort de l'ensemble des témoignages, des finissants de 1981 comme de 1991, que le développement du tourisme passe par la professionnalisation. et que le défi de la régionalisation se vit à l'ère de la mondialisation.

En effet, il importe qu'une formation en tourisme développe, en plus des connaissances et des compétences propres au domaine, la polyvalence, la capacité d'adaptation et l'auto-formation, en privilégiant des activités pratiques d'intégration à l'industrie en cours de formation.

Ces conclusions rejoignent les orientations confirmées par le nouveau programme de
Techniques de tourisme"ll. Conçu selon une approche globale, ce programme permet une formation polyvalente et tient compte principalement des besoins du marché du travail, des finalités et buts généraux de la formation professionnelle au collégial.

C'est dans cet esprit que le Cégep de Matane devient le premier établissement collégial à offrir le programme de Techniques de tourisme selon la formule de l'alternance travailétudes. Ainsi, dès 1995, nos finissants auront acquis de neuf à douze mois d'expérience pratique en occupant différents postes dans trois secteurs de l'industrie touristique.

Merci à nos collaborateurs pour leur participation empressée et la qualité de leurs témoignages. Ceux-ci illustrent très bien toute l'importance de la dimension humaine dans l'industrie du tourisme.

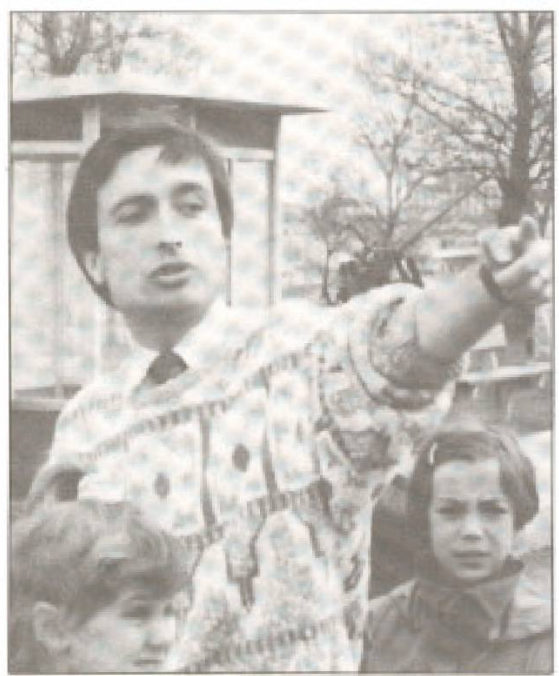

Michel Hébert

\section{Formation et action}

Michel HÉBERT est directeur du tourisme à l'Office du tourisme et des congrès de Rimouski. Il a complété un baccalauréat en gestion et intervention touristiques à l'Université du Québec à Montréal

En tant que directeur du tourisme, je travaille tant au niveau du développement que de la promotion du tourisme d'agrément rimouskois. Dans les faits, cela s'est progressivement traduit au cours des sept dernières années par la prise en charge totale ou partielle des mandats suivants: l'encadrement et la formation du personnel d'accueil ou des guides accompagnateurs; la production de brochures et de dépliants promotionnels; le support technique aux promoteurs et gestionnaires de sites, attraits et services touristiques; la coordination des présences del'Office aux salons de tourisme d'agrément; les relations publiques tant dans le milieu rimouskois qu'avec les chroniqueurs en tourisme; l'établissement de liens plus étroits avec les intervenants de l'industrie du voyage, etc. De plus, mon intérêt pour l'informatique m'a amené à contribuer à la formation du personnel de l'Office dans le cadre du processus d'informatisation des diffërentes tâches.

Pour moi, la principale force du programme de gestion et intervention touristiques de l'UQAM est de former des généralister conscients des multiples facettes de notre industrie. Sanscette formation, la compréhension du fonctionnement de l'industrie touristique aura exigé passablement de temps et je n'aurais pu être efficace dès le départ.

Ainsi, une bonne partie des connaissances transmises dans les cours d'administration me servent régulièrement à préparer les budgets de differents projets; les cours de méthodologie de recherche et de statistiques s'avèrent utiles dans la compréhension de rapports de consultants et dans la préparation du rapport saisonnier sur la fréquentation du bureau d'information touristique. Etant donné que plusieurs de nos actions doivent être réalisées dans un court laps de temps, avec des ressources limitées, un cours comme planification et contrôle de projets est utile plus d'une fois 


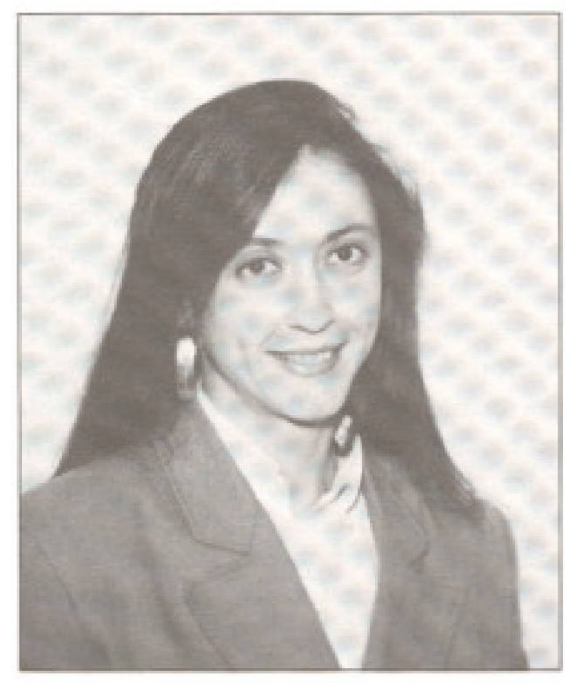

Nathalie Tuboeuf

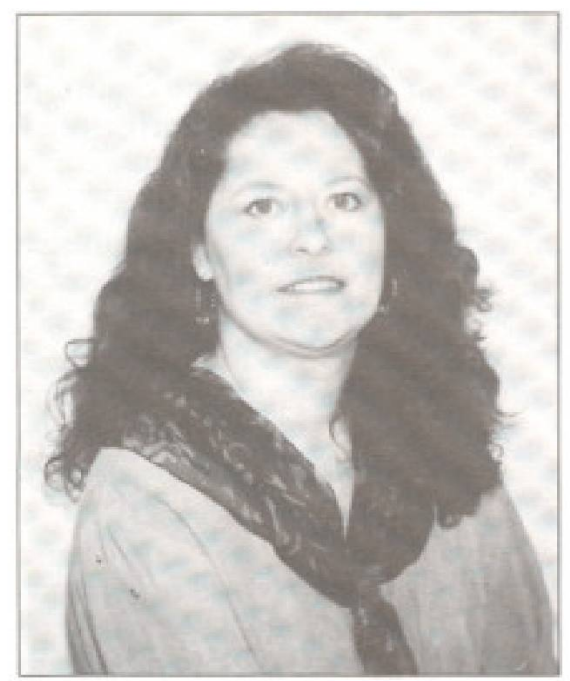

Edith Thibault par année. Et les exemples peuvent contnuer ainsi...

Avec le recul d'une décennie (oufl), les étudiants qui, comme moi, ont pu marier études et implication dans le milieu touristique pendant leur baccalauréat ont phus de chances de se trouver un emploi.

Nathalie TUBOEUF est directrice générale du Comité d'aide au développement de la collectivité (CADC) de Gaspé-Nord à Sainte-Anne-desMonts. Elle détient une maîtrise en tourisme de l'École supérieure de tourisme et d'hôtellerie de l'Université d'Angers, France

Comme son nom l'indique, le CADC a pour mission d'apporter un soutien au développement des collectivités en difficultés socio-économiques. Le territoire desservi par cet organisme couvre la MRC DenisRiverin et une partie de la MRC Côte-deGaspé. Il s'étend de Les Capucins jusqu’à Murdochville: un vaste territoire.. pour une large implication.

Pour les promoteursde projets de développement, le soutien se caractérise par un apport d'informations, de conseils, et éventuellement d'une participation financière par le biais de programmes spécifiques.

Pour les différents organismes de développement en place, ce soutien se traduit par un effort de concertation et de partenariat.

Enfin, de manière plus générale, et en relation avec cette philosophie, cesupport se définit aussi actuellement par l'élaboration d'une planification stratégique de développement pour la collectivité.

L'équipe de travail étant généralement très petite, être en charge d'une CADC revient ainsi à ètre le chef d'orchestre et.. l'orchestre! Cela requiert une grande polyvalence en termes de connaissances et d'aptitudes. Connaissances de l'ensemble des secteurs socio-économiques, aptitudes en termes de leadership, de communication et d'adaptation aux différentes situations rencontrées.

Dans ce contexte, en quoi une maitrise en tourisme peut-elle m'être utile? Cette formation en tourisme s'avère des plus profitables, et je ne saurais me passer de ce qu'elle m'a apporté. Deux raisons essentielles à cela. La première est reliée au contenu même de la formation suivie en France. Ia seconde, au contexte de développement gaspésien, et plus particulièrement nord-gaspésien.

Le programme de second cycle offert par l'Université d'Angers est basé sur un apprentissage polyvalent: caractérisation de produits, principes de développement, aménagement du territoire, marketing, communication, etc. Ce programme a également pour particularité de faire vivre aux étudiants deux expériences de terrain, durant respectivement trois et six mois, et, à l'issue, de leur faire produire un rapport derecherche et un mémoire. J'ai mené ces deux expériences au Québec, l'une portant sur le tourisme d'aventure sur la CôteNord et l'autre sur les communautés autochtones du Québec.

Il fautaussi dire que le développement dela Gaspésie est particulièrement axé sur le tourisme. Voic l'autre raison pour laquelle ma formation $m^{7}$ est indispensable. Le développernent du secteur touristiquea été réccemment mis de l'avant par le Conseil régional de concertation et de développement Gaspésie/Iles-de-la-Madeleine (CRCD) dans le cadre de sa planification stratégique. Les différents CADCetautres organismes de développement économique présents dans le territoire perçoivent également ce secteur comme prioritaire. II faut donc que le milieu, maiture d'oeuvre, s'implique. Je participais, il y a peu, a la rencontre du comité provisoire de promotion touristique de la MRC de DenisRiverin. Dansnotreterritoire, une évidence apparaît clairement: notre produit manque destructure, de cohésion, et n'exerce pas à l'heure actuelle un attrait majeur pour la clientèle touristique de passage. Mis en place il y a quelques mois à l'initiative de la

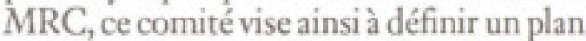
d'action préconisant des activités promotionnelles à mener. Il faut aussi structurer l'offre touristique en fonction des attraits du territoire et des attentes des clientèles potentielles, et vite! Dans notre collectivité, là est le défi.

Edith THIBAULT est conseillêre en développement pour la municipalité régionale de comtể de Denis-Riverin à Sainte-Anne-des-Monts. Ellepossède un baccalauréat en gestion et intervention touristiques de l'Université du Québec à Montréal

Je travaille pour la MRCde Denis-Riverin et $85 \%$ de mes interventions se font dans 


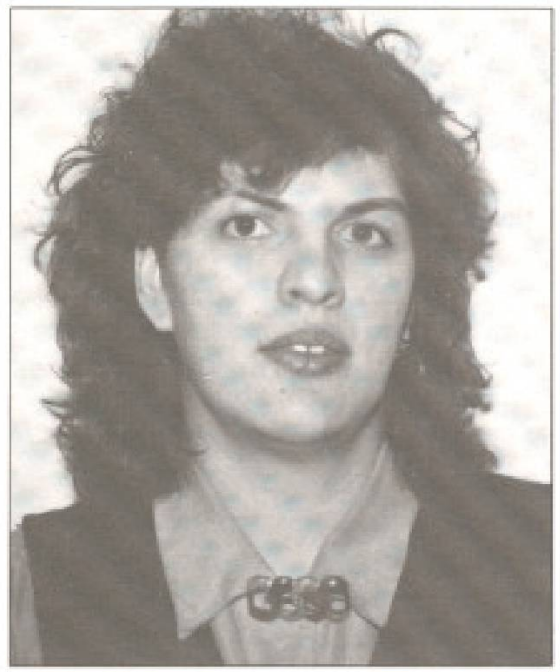

Lise Rioux

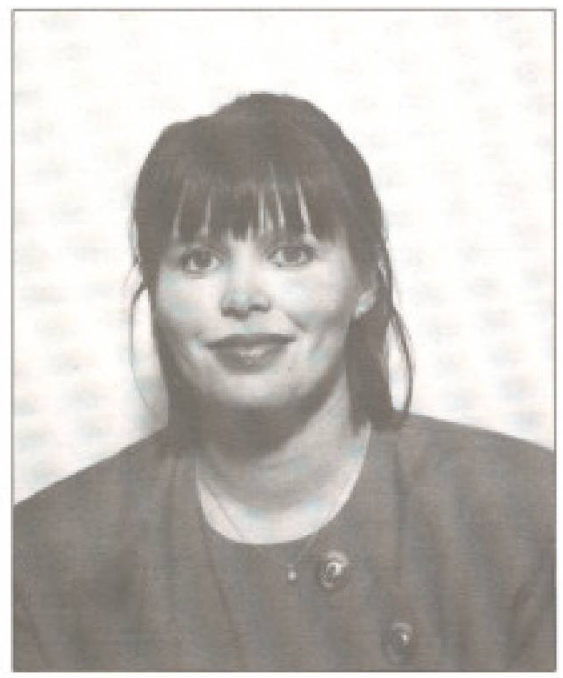

Gina Bernier le secteur touristique. Mon mandat consiste à planifier le développement sur le territoire, orchestrer la promotion touristiqueen collaboration aveclesintervenants du milieu et animer ce secteur en ce qui a trait à la formation, l'accueil et la promotion. Je collabore étroitement avec le CADC de Gaspé-Nord pour la réalisation d'une planification stratégique, laquelle devrait nous donner des assises solides pour consolider et positionner notre produit au cours des prochaines années. Enfin, je participe, par uneaide technique, à l'élaboration de projets deplans d'affaires avecdes intervenants touristiques désireux d'améliorer leurs produits ou services et qui sont à la recherche de financement. Mon travail est très varié puisque je touche à plusieurs facettes de l'industrie touristique.

La formation acquise m'a ouvert la porte pour entrer dans le grand et, à la fois, et petitmonde du tourisme et la spécialisation en planification me convenait parfaitement. Mon baccalauréat m'a permis d'aller chercher une base d'informations suffisante pour accepter de très beaux défis et mon désir d'avancer et progresser dans cette industrie a fait le reste. Aujourd'hui, avec un certain recul, je peux dire que je n'ai jamais cessé ma formation puisque j'apprends tous les jours et, avec le temps, j'acquiers une confiance et des compétences qui me permettent de participer pleinement aux efforts de développement de ma région.

L'expérience aidant, j'ai compris que le développement ça ne se fait pas en un jour, et ma patience s'est beaucoup armée face aux nombreux délais auxquels on doit souvent faire face. Lorsque l'on arrive fraîchement diplômée, on voudrait que tout se fasse rapidement mais la réalité est tout autre car il faut savoir composer avec des facteurs sur lesquels nous n'avons pas beaucoup d'emprise.

Lise RIOUX est coordonnatrice du Comité de développement de Bic-SaintFabien. Elle détient un diplôme d'études collégiales en tourisme du Collège de Matane

Issu de la complicitéde deux municipalités, le mandat du comité réside dans le développement, l'animation et la promotion de son territoire. Sept années d'implication dans les domaines aussi variés que le développement, l'accueil, l'animation et la gestion m'ont permis d'accéder à la direction de cette corporation. Mon mandat de travail consiste principalement à: favoriser sur le territoire un ensemble d'activités et de services diversifiés et de qualité mettant en valeur les potentiels économiques et touristiques; assurer l'accueil et l'information sur le territoire; créer des outils pour promouvoir notre territoire comme zone dedestination court-séjour et dereprésenter celui-ci lors d'événements spéciaux (salon, tour de familiarisation, média); représenter les intervenants du milieu auprès de diverses instances gouvernementales ou autres; superviser le personnel nécessaire à la mise en application de ces activités; administrer la corporation.

Ma formation en Techniques du tourisme me guide depuis mes premiers pas dans l'industrie. Elle est une référence importante que l'on doit mettre à jour et spécialiser.

La connaissance des clientèles et l'application des techniques d'accueil, d'animation, d'organisation et de communication constituent la base de tout projet.

Outre la formation, il est important d'accroittre nos connaissances selon les objectifs de l'entreprise, de se spécialiser dans le domainedelagestion et del'administration, de s'impliquer dans différents réseaux du domaine, d'être informé de l'évolution de l'industrie et d'être aux aguets de tous changements reliés à notre environnement.

Gina BERNIER est directrice de l'agence de voyages Club voyages Bravo. Elle a complété un diplôme d'études collégiales en tourisme au Collège de Matane

La fonction de directrice d'agence de voyages touche essentiellement la vente, le marketing et les relations publiques.

Plus spécifiquement, les tảches consistent dansl'organisation et/ou la vente de forfaits divers (déjà constitués et sur mesure, individuel ou pour les groupes), la vente de titres detransport, la promotion de l'agence et de ses produits, la comptabilité des dossiers des clients et l'administration générale du bureau.

J'ai travaillé d'abord en développement touristique pour le comité organisateur des fêtes Matane 1534-1984. Si je me suis retrouvée à l'emploi d'une agence de voyages 
trois ans après avoir terminé ma formation en tourisme, c'est un peu par un concours de circonstances.

Mon employeur me trouvait assez dégourdie et vive d'esprit puisque j'avais amplement eu l'occasion d'acquérir, tout au long de ma formation, des connaissances générales en ce qui a trait à la géographie, aux attractions touristiques, etc., ainsi qu'une bonne idée du fonctionnement d'une agence de voyages.

Les activités intégrées aux cours de tourisme, liéesà des événementsde type salons ou congrès, m'ont permis de développer des aptitudes pour le contact avec les gens et pour créer rapidement un climat de confiance.

Deplus, ayant la chance de faire partie d'un regroupement important tel que Club voyages, des sessions de formation sur mesure nous permettent (surtout en région) d'être à la fine pointe des dernières tendances de l'industrie du voyage.

De nos jours, avoir sa propre entreprise exige énormément de ténacité, de volonté, de persuasion, de connaissances et surtout de contacts dans différents domaines.

Isabelle MICHAUD est responsable de l'accueil au Village de vacances Léo Lagrange en Corse. Elle détient un diplồme d'études collégiales du Collège de Matane

De façon gểnérale, mes tầches consistent à adapter les techniques d'accueil selon les différentesclientèles (Français, Allemands, Italiens, familiale ou de groupe), à superviser les hôttesses et à tenter de créer un esprit d'équipe et de service, à élaborer et à mettre à la disposition des gens des outils qui les aideront à mieux vivre leurs vacances (manuel de référence sur les activités offertes). Ma plus grande responsabilité demeure quand même de voir à ce que le client soit plus que satisfait de l'accueil et de l'information qu'il reçoit.

Ma formation en tourisme me sert tous les jours. Quand on agit instantanément face a une situation professionnelle, cela signifie qu'on a retenu quelque chose de notre formation. Alors, on se découvre, on réalisequ'on possède des compétences pour la vente, l'organisation d'événements ou encore la facilité d'établir des contacts lors d'un accueil ou de l'animation de groupe, de la gestion de plaintes, etc. De plus, les techniques de rédaction de documents administratifs et les principes de base de la gestion m'ont permis de gravir un ếchelon de plus.

Une des choses qu'on m'a enseignée est de bien connaiture les différentes sources et les outils à notre disposition car personne ne possède toutes les connaissances. Peu importe le niveau de formation que l'on possède, nous devons accepter le fait que celle-ci n'est jamais terminée.

Pour offrir un bon accueil, il faut d'abord et avant tout se mettre à la place du client et c'est une des raisons pour lesquelles j'ai choisi d'acquérirmonexpérience en France. Si j'ai bien compris, les années à venir visent à conquérir le coeur des Européens; alors, nous devons tout d'abord nous rendre chez eux pour étudier leurs besoins et ensuite tenter d'y répondre selon notre culture. On ne peut pas tout apprendre dans les livres et, fort heureusement, c'est un des plaisirs de la vie.

Jean-Marc MAJOR est agent d'animation et d'information au Musée acadien du Québec à Bonaventure. II possède un diplôme d'études collégiales du Collège de Matane.

Comme agent d'animation et d'information, ma tẩche concerne la diffusion: faire connaître et apprécier la réalité du musée à l'extếrieur pour sensibiliser d'éventuels visiteurset fairevivreà ceux qui seprésentent au musée une page d'histoire et une époque, à l'aide de la collection d'artefacts.

Ma plus grande responsabilité est de voir à ceque touslesvisiteurs trouventleurcompte lors de leur passage au musée. Mon principal rôle consiste à les rejoindre et, par la suite, à leur faire vivre la réalité du musée. Pour ce faire, il fautimpliquer toutel'équipe du musée, principalement l'équipe d'été, qu'il faut former, motiver, évaluer.

De ma formation en tourisme, j'ai retenu deux points qui, pour moi, sont fondamentaux dans l'exercice de mon travail: 1 l'importance de bien connaître son produit; et 2- la capacitéde bien communiquer ses connaissances. J'en fais la règle d'or de ma profession et la passe à mon tour à ceux et celles qui sont appelés à travailler comme animateur au musée.

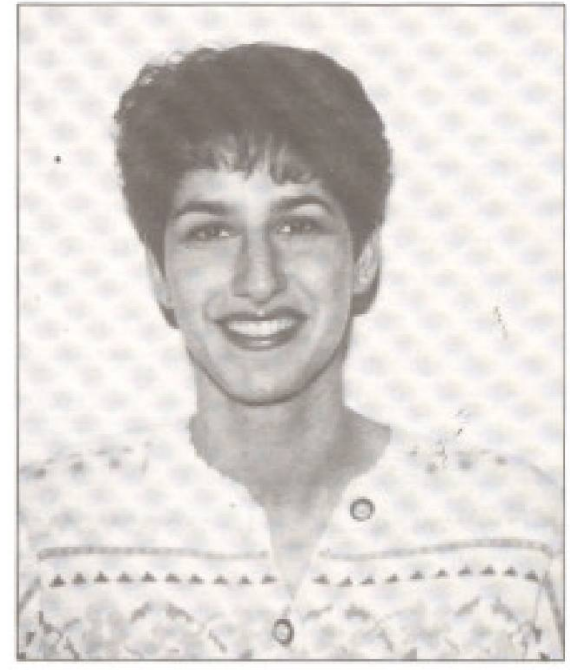

Isabelle Michaud

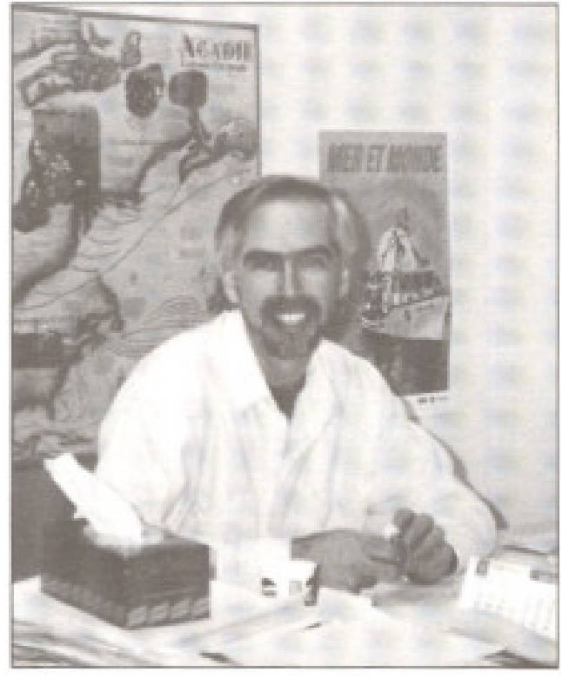

Jean-Marc Major 
J'entends encore mes professeurs dire que pour travailler en tourisme on doit tout connaître: l'histoire, la géographie, l'économie, la politique, les curiosités, etc., de son monde. Je réalise à chaque jour comment celà est vrai, comment le visiteur en pays nouveau cherche à connaître et à comprendre ce qu'il découvre; tout l'interpelle. Il faut tendre à tout connaître ou du moins accepter d'être en apprentissage perpétuel pour être un bon intervenant touristique. $\mathrm{Ma}$ formation en tourisme m'a inculqué quelques techniques utiles - il faut bien le dire - maisl'ouverture qu'elle a suscitée chez moi m'est encore plus précieuse.

\section{$\cdots \cdots$}

\section{Visions de la Gaspésie de I'an 2000}

Les responsables du développement touristique régional n'ont pas manqué de regarder l'avenir malgré leurs responsabilités et leurs nombreuses tẩches quotidiennes. Nous leur laissons la parole à ce propos, en soulignant leur confiance dans l'avenir d'une part mais aussi leur sens du réel et du possible.

*Les voyagistes qui s'attardentau réceptif auraient beaucoup de travail à faire, notamment quant au tourisme à thème, soit: la thalassothérapie, les parcs nationaux, le cachet historique et typique de la Gaspésie. Il serait aussi facile de viser les gens d'affaires pour leur offrir la Gaspésie en tant que voyage de motivation, $*$ Gina BERNIER

*Comme la Côte d'Azur, la Guadeloupe et la Corse, la Gaspésie vit de la mer et du tourisme! Le fait est simple, il suffit de répondre aux besoins des gens et ici c'est possible car ceux-ci demandent espace, nature, sourire et simplicité. Il estimpossible de demander à notre jeune province de raconter 1000 ans d'histoire, mais quoi de plus beau que de faire parler notre paysage et notre culture dans la langue de nós invités!» Isabelle MICHAUD

*Paradoxalement si je trouve la Gaspésie touristique actuelle dynamique, c'est plutôt une inquiếtude que m'inspire la Gaspésie touristique de l'an 2000. Les politiciens de la Gaspésie ont placé en tête de liste, pour le développement de la région, le tourisme. L'inquiétude que $j$ 'ai est la suivante: sil l'économie basée sur les matières premières, telles le poisson, la forêt, les mines, etc., périclite, et si le tourisme se développe davantage, n'y at-il pas danger de réduire la Gaspésie ằ un lieu de résidence d'été? Bien sûr, j'ai à coeur le développement touristique, mais pas au détriment des autres composantes fondamentales de l'économie. Le tourisme à lui seul peut-il faire vivre convenablement la Gaspésie? Je ne sais pas, mais la perspective d'une Gaspésie bondée pendant trois mois d'été et désertée pendant neuf mois le reste de l'année, me fait penser à une grossesse dangereuse. De quel genre de filles et de fils la Gaspésie accouchera-t-elle après neuf mois de léthargie dans un pays endormi? Seront-ils assez vigoureux pour bien recevoir la visite? J'espère que mon inquiétude est non fondée.» Jean-Marc Major

*La Gaspésie a su conquérir plus d'une génération de plaisanciers. L'offre des autres régions se raffine et est de plus en plus attrayante. La Gaspésie devra être plus agressive pour conserver sa part de marché et devra s'adapter à cette clientèle plus critique et mieux organisée.» Lise RIOUX

«Le Bas-Saint-Laurent est maintenant rendu à une étape où l'on doit consolider les sites et les attraits, aider à la naissance de nouveaux attraits dans la mesure où ceux-ci complètent l'offre actuelle (notamment au niveau de leur thématique), développer le produit hiver, autant pour la clientèle québécoise que pour la clientèle internationale, et tenter de résoudre le sempiternel problème d'étalement de la saison au printemps et surtout à l'automne.» Michel HÉBERT

*Vue sous l'angle des vacances, la Gaspésie représente pour nombre de Québécois une destination de choix. Ils en ont déjà fait le tour, ou rêvent de le faire un jour, un été, en quelques étapes... Vu sous l'angle de la vie quotidienne, au fil des saisons, le portrait de la Gaspésie n'est plus toujours le même. La Gaspésie, c'est loin, c'est pauvre... Lorsque l'on m'a offert de venir travailler à SainteAnne-des-Monts pour le Comité d'aide au développement de la collectivité de Gaspé-Nord (CADC), j'avoue avoir eu un temps d'hésitation. Après toutes ces années passées à Québec... Aujourd'hui, je sais que ce coin de pays constitue une richesse extraordinaire, et je crois en lui. Car la Gaspésie prise dans son ensemble, tout comme certaines autres régions du
Québec, est appelée à développer considérablement son potentiel touristique et à capter les marchés réceptifs, comme les marchés européens. Pour elle aussi l'enjeu est grand, car la concurrence mondiale se renforce. La Gaspésie ne doit pas s'attarder sur ses acquis antérieurs et se dire qu'il lui suffit de demeurer un circuit touristique de choix (mais pour combien de temps encore?). Elle doit prendre sa place, consolider ses acquis, miser sur de nouveaux produits, et se tourner vers des marchés en croissance. Et cela ne se situe pas seulement d'un point de vue touristique. Notre économie, ce sont aussi les ressources naturelles, et des domaines innovateurs comme la technologie, pourquoi pas? Ce n'est pas uniquement le développement d'un secteur d'activité comme le tourisme qui est en cause, c'est la survie d'une région. Pour réussir, il nous faut $y$ croire, croire en notre richesse et se donner les outils pour la mettre en valeur.» Nathalie TUBOEUF

*Ma vision du produit touristique de la Gaspésie? Nous avons un produit dynamique et très varié. Tant et aussi longtemps que l'on se développera en préservant l'authenticité des villes et villages et l'accueil de sa population, la Gaspésie préservera son charme et sa notoriété qu'elle affiche fièrement. Beaucoup d'efforts sont faits dans plusieurs localités pour implanter de nouveaux produits et toute cette effervescence fait qu'aujourd'hui les touristes se retrouvent devant une brochette d'activités bien garnie, qui répond à plusieurs types de clientèles. Il y a toujours des améliorations à apporter ici et là sur certains produits et services mais, comme "Paris ne s'est pas fait en un jour ${ }^{n}$, la Gaspésie saura, à son tour, se bấtir un produit fort et distinctif pour conserver sa place parmi les meilleures destinations-vacances au Québec. Ilya tout un monde qui travaille audéveloppement touristique de la région et l'union de ces efforts est, à mon point de vue, porteur d'avenir. C'est pourquoi j'ai pleinement confiance au développement progressif du produit touristique gaspésien. Venez nous visiter, on vous attend, $\gg$ Edith THIBAULT

\section{NOTES ET RÉFÉRENCES}

(1) Gouvernement du Québec, ministẻre de l'Enseignement supérieur, Servicé du développement des programmes, 414.01 - Techniques de tourisme, 1992 